\title{
Algebras of Borel measurable functions
}

\author{
by
}

\author{
Michał Morayne (Wrocław)
}

\begin{abstract}
We show that, for each $0<\alpha<\omega_{1}$, in the $\alpha$ th class in the Baire classification of Borel measurable real functions defined on some uncountable Polish space there is a function which cannot be expressed as a countable union of functions which are (on their domains) in the $\alpha$ th class in Sierpiński's classification. This, in particular, solves positively a problem of Kempisty who asked whether for $1<\alpha<\omega_{1}$ the $\alpha$ th Baire and Sierpiński classes are different. We also show that, for every $\alpha$, in the $\alpha$ th class of Sierpiński's classification there is a function which cannot be expressed as a countable union of functions each of which is on its domain in one of the two $\alpha$ th classes of Young's classification (we refer here to the classical numbering of Baire's, Young's and Sierpiński's classes and not to the one used in the paper).
\end{abstract}

1. Introduction. In $[\mathrm{CM}]$ and $[\mathrm{CMPS}]$ the following diagram was considered:

$\mathbf{B}_{\alpha}$

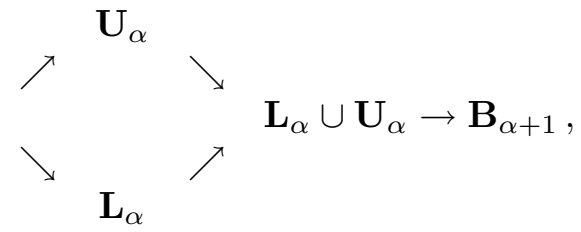

where $\mathbf{B}_{\alpha}$ is the $\alpha$ th class in the Baire classification of real functions defined on $[0,1]$ and $\mathbf{L}_{\alpha}$ and $\mathbf{U}_{\alpha}$ are the classes of limits of, respectively, nondecreasing and nonincreasing sequences of functions from $\mathbf{B}_{\alpha}$; the arrows stand for proper inclusions. It was shown there that in every class of (1) there is a function which cannot be expressed as a union of countably many partial functions from lower classes. In the present paper, considering the algebra $\mathbf{L}_{\alpha}+\mathbf{U}_{\alpha}$ of all algebraic sums of functions from $\mathbf{L}_{\alpha}$ and $\mathbf{U}_{\alpha}$, we add to (1) the following diagram (cl stands for closure in the uniform convergence

1991 Mathematics Subject Classification: Primary 26A21.

Supported in part by KBN grant 2-1054-91-01. 
topology):

$$
\mathbf{L}_{\alpha} \cup \mathbf{U}_{\alpha} \rightarrow \mathbf{L}_{\alpha}+\mathbf{U}_{\alpha} \rightarrow \operatorname{cl}\left(\mathbf{L}_{\alpha}+\mathbf{U}_{\alpha}\right)=\mathbf{B}_{\alpha+1}
$$

(the equality in (2) was proved by Sierpiński in $\left[\mathrm{S}_{2}\right]$ for $\alpha=0$ and the proof remains the same for all $\left.\alpha<\omega_{1}\right)$. In fact, we consider algebras of bounded functions and then the diagram (2) gets more subtle (b stands for the bounded functions in the given class):

$$
\begin{aligned}
\mathrm{bL}_{\alpha} \cup \mathrm{b} \mathbf{U}_{\alpha} \rightarrow \mathrm{b} \mathbf{L}_{\alpha} & +\mathrm{b} \mathbf{U}_{\alpha} \\
& \rightarrow \operatorname{cl}\left(\mathrm{b} \mathbf{L}_{\alpha}+\mathrm{b} \mathbf{U}_{\alpha}\right) \rightarrow \mathrm{b}\left(\mathrm{cl}\left(\mathbf{L}_{\alpha}+\mathbf{U}_{\alpha}\right)\right)=\mathrm{b} \mathbf{B}_{\alpha+1} .
\end{aligned}
$$

Again we show that in every class displayed in (3) there exists a function which cannot be expressed as a sum of countably many partial functions from lower classes. This, in particular, implies that the second inclusion from (2), $\mathbf{L}_{\alpha}+\mathbf{U}_{\alpha} \subset \operatorname{cl}\left(\mathbf{L}_{\alpha}+\mathbf{U}_{\alpha}\right)=\mathbf{B}_{\alpha+1}$, is proper. This solves a problem of Kempisty [Ke] (for $\alpha=0$ the inclusion was shown to be proper by Sierpiński $\left.\left[\mathrm{S}_{1}\right]\right)$.

We work in a more general setting enabling us to obtain, for example, analogous results for functions measurable with respect to the projective classes $\Sigma_{n}^{1}$.

2. Notation, definitions and basic facts. We use standard set-theoretical notation. $\mathbb{N}$ is the set of positive integers, $\mathbb{R}$ the set of reals, and $P(A)$ the family of all subsets of a set $A$. If $A$ is fixed and $\mathcal{A} \subseteq P(A)$ then $\mathcal{A}^{\mathrm{c}}=\{A \backslash B: B \in \mathcal{A}\}$. $\mathcal{A}_{\delta}$ will stand for all countable intersections of elements of $\mathcal{A}$. A family $\mathcal{A} \subseteq P(A)$ is a partition of $A$ if $\cup \mathcal{A}=A$ and for all $X, Y \in \mathcal{A}, X \neq Y$, we have $X \cap Y=\emptyset$. If $\mathcal{A} \subseteq P(A)$ and $X \subseteq A$, then $\mathcal{A} \mid X=\{Y \cap X: Y \in \mathcal{A}\}$. We denote by $r(\mathcal{A})$ the ring of sets generated by $\mathcal{A}$, i.e. the smallest family containing $\mathcal{A}$ and closed under taking complements and finite unions. Suppose that $\mathcal{A} \subseteq P(A)$ is a family of sets. We say that $\mathcal{A}$ is a $\sigma$-class if $\{\emptyset, A\} \subseteq \mathcal{A}$ and $\mathcal{A}$ is closed under finite intersections and countable unions. If $\mathcal{A} \subseteq P(A)$, then we denote by $\mathcal{A}^{\prime}$ the minimal $\sigma$-class containing $r(\mathcal{A})$. The symbol $\chi_{A}$ will denote the characteristic function of $A$. The domain of a function $f$ will be denoted by $\operatorname{dom} f$ and its range by $\operatorname{Rg} f$. If $A$ and $B$ are sets, then ${ }^{A} B$ is the set of all functions with domain $A$ and range contained in $B$. If $f \in{ }^{A} B$ and $C \subseteq A$, then $f \mid C$ denotes the restriction of $f$ to $C$. We write ${ }^{A} B$ for the set of all partial functions from $A$ to $B$, i.e. ${ }^{A} B=\left\{f \in{ }^{C} B: C \subseteq A\right\}$. Let $f$ be a real function defined on some set $A$; then $\inf f=\inf \{f(x): x \in A\}$, $\sup f=\sup \{f(x): x \in A\}$ and if $f$ is bounded $\|f\|=\sup |f|$. If $\mathcal{H}$ is any

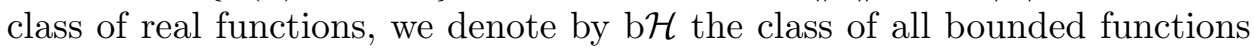
from $\mathcal{H}$, and by $\operatorname{cl} \mathcal{H}$ the class of all uniform limits of functions from $\mathcal{H}$. For $\mathcal{G} \subseteq{ }^{Z} \mathbb{R}$ and $\mathcal{H} \subseteq{ }^{Z} \mathbb{R}$ let $\mathcal{G}+\mathcal{H}=\{g+h: g \in \mathcal{G}$ and $h \in \mathcal{H}\}$. Let $A \subseteq \mathbb{R}$. 
We denote by $V B(A)$ the family of all real functions of bounded variation on $A$, and by $C(A)$ the continuous functions on $A$.

We write $\mathcal{N}$ and $\mathcal{C}$ for the spaces ${ }^{\mathbb{N}} \mathbb{N}$ and ${ }^{\mathbb{N}}\{0,1\}$, respectively, with the product topology. The first space is homeomorphic to the irrational numbers and the second to the Cantor set.

Let $Z$ be any set. Let $\mathcal{F} \subseteq Z_{\mathbb{R}}$ and $\mathcal{G} \subseteq Z_{\mathbb{R}}$. We denote by $\operatorname{dec}(\mathcal{F}, \mathcal{G})$ the least cardinal $\kappa$ such that for every $f \in \mathcal{F}$ one can find a family $\left\{g_{\alpha}\right.$ : $\alpha<\kappa\} \subseteq \mathcal{G}$ such that $\left\{\operatorname{dom} g_{\alpha}: \alpha<\kappa\right\}$ is a partition of $Z$ and $f=\bigcup\left\{g_{\alpha}\right.$ : $\alpha<\kappa\}$. We shall only use this definition when it makes sense, i.e., when such subfamilies of $\mathcal{G}$ exist.

Suppose $\mathcal{A}$ is a $\sigma$-class. We denote by $\overline{\mathbf{M}} \mathcal{A}$ the family of all functions $f \in Z_{\mathbb{R}}$ such that $f^{-1}((-\infty, c)) \in \mathcal{A}$ for every $c \in \mathbb{R}$. Similarly, $\underline{\mathbf{M}} \mathcal{A}$ is the family of all $f \in{ }^{Z} \mathbb{R}$ such that $f^{-1}((c, \infty)) \in \mathcal{A}$ for every $c \in \mathbb{R}$. Note that $f \in \underline{\mathbf{M}} \mathcal{A}$ if and only if $-f \in \overline{\mathbf{M}} \mathcal{A}$. We put $\mathbf{M} \mathcal{A}=\underline{\mathbf{M}} \mathcal{A} \cap \overline{\mathbf{M}} \mathcal{A}$. We denote by $\mathbf{M}_{B} \mathcal{A}, \underline{\mathbf{M}}_{B} \mathcal{A}$ and $\overline{\mathbf{M}}_{B} \mathcal{A}$ the functions from $\mathbf{M} \mathcal{A}, \underline{\mathbf{M}} \mathcal{A}$ and $\overline{\mathbf{M}} \mathcal{A}$, respectively, for which $\operatorname{Rg} f \subseteq B$. Note that if $\mathcal{A}$ is a $\sigma$-class and $B$ is closed then $\mathbf{M}_{B} \mathcal{A}, \underline{\mathbf{M}}_{B} \mathcal{A}$ and $\overline{\mathbf{M}}_{B} \mathcal{A}$ are complete metric spaces (in the uniform convergence topology) and the same is true for $\mathrm{bM} \mathcal{A}, \mathrm{bM} \mathcal{A}$ and $\mathrm{b} \overline{\mathbf{M}} \mathcal{A}$. Let $R \underline{\mathbf{M}}_{B} \mathcal{A}=\bigcup\left\{\underline{\mathbf{M}}_{B}(\mathcal{A} \mid X): X \in P(Z)\right\}, R \overline{\mathbf{M}}_{B} \mathcal{A}=\bigcup\left\{\overline{\mathbf{M}}_{B}(\mathcal{A} \mid X): X \in\right.$ $P(Z)\}, R\left(\underline{\mathbf{M}}_{B} \mathcal{A}+\overline{\mathbf{M}}_{B} \mathcal{A}\right)=\bigcup\left\{\underline{\mathbf{M}}_{B}(\mathcal{A} \mid X)+\overline{\mathbf{M}}_{B}(\mathcal{A} \mid X): X \in P(Z)\right\}$ and $R\left(\operatorname{cl}\left(\mathrm{b} \underline{\mathbf{M}}_{B} \mathcal{A}+\mathrm{b} \overline{\mathbf{M}}_{B} \mathcal{A}\right)\right)=\bigcup\left\{\mathrm{cl}\left(\underline{\mathrm{bM}}_{B}(\mathcal{A} \mid X)+\mathrm{b} \overline{\mathbf{M}}_{B}(\mathcal{A} \mid X)\right): X \in P(Z)\right\}$.

We use standard notation from Descriptive Set Theory. For example, $\Sigma_{\alpha}^{0}$ $\left(\Pi_{\alpha}^{0}\right)$ denotes the $\alpha$ th additive (multiplicative, resp.) class in the hierarchy of Borel sets, and $\Sigma_{n}^{1}$ is the $n$th projective class in the hierarchy of projective sets.

For $X$ a Polish space and $\alpha<\omega_{1}$, let $\mathbf{B}_{\alpha}(X)=\left\{f \in \mathbb{R}_{\mathbb{R}}: f^{-1}(G) \in\right.$ $\Sigma_{1+\alpha}^{0}(X)$ for each $G$ open in $\left.\mathbb{R}\right\}$. If $X=\mathbb{R}$ we write briefly $\mathbf{B}_{\alpha}(\mathbb{R})=\mathbf{B}_{\alpha}$. We have $\mathbf{B}_{\alpha}(X)=\mathbf{M} \Sigma_{1+\alpha}^{0}(X)$. We also write $\mathbf{L}_{\alpha}(X)$ and $\mathbf{U}_{\alpha}(X)$ to denote $\underline{\mathbf{M}} \Sigma_{1+\alpha}^{0}(X)$ and $\overline{\mathbf{M}} \Sigma_{1+\alpha}^{0}(X)$, respectively. For $X=\mathbb{R}$ we write $\mathbf{L}_{\alpha}(\mathbb{R})=\mathbf{L}_{\alpha}$ and $\mathbf{U}_{\alpha}(\mathbb{R})=\mathbf{U}_{\alpha}$. Obviously, $\mathbf{L}_{0}(X)$ and $\mathbf{U}_{0}(X)$ are the classes of lower and upper semicontinuous functions on $X$ with values in $\mathbb{R}$.

R e m a r k. In the classical notation the class $\mathbf{B}_{\alpha}$ for $\alpha<\omega$ and $\mathbf{B}_{\alpha+1}$ for $\alpha \geq \omega$ is called the $\alpha$ th class in the Baire classification and the classes $\mathbf{L}_{\alpha}$, $\mathbf{U}_{\alpha}\left(\mathbf{L}_{\alpha}+\mathbf{U}_{\alpha}\right)$ for $\alpha<\omega$ have the number $\alpha+1$ and for $\alpha \geq \omega$ the number $\alpha$ in Young's (Sierpiński's, resp.) classification (compare for instance [L]).

We say that a class $\mathcal{A}$ has the reduction property if for any $A, B \in \mathcal{A}$ there are $A^{*}, B^{*} \in \mathcal{A}$ such that $A^{*} \subseteq A$ and $B^{*} \subseteq B, A^{*} \cap B^{*}=\emptyset$ and $A^{*} \cup B^{*}=A \cup B$. Note that if $1<\alpha<\omega_{1}$, then $\Sigma_{\alpha}^{0}$ has the reduction property. The same is true of $\Sigma_{n}^{1}, n \in \mathbb{N}$. Moreover, if $Z$ is zero-dimensional, then $\Sigma_{1}^{0}(Z)$ also has the reduction property.

Following the idea used in $[\mathrm{Mo}]$ we deal in this paper with a certain fixed 
family $\mathcal{T}$ of Polish spaces such that:

(i) if $X \subseteq Z \in \mathcal{T}$ and $X$ is a closed subset of $Z$, then also $X \in \mathcal{T}$;

(ii) $\mathcal{T}$ is closed under finite Cartesian products;

(iii) $\mathcal{N}, \mathbb{R} \in \mathcal{T}$.

As in $[\mathrm{Mo}]$ the idea is to include in $\mathcal{T}$ any Polish space one wants to consider.

Now assume that to each $Z \in \mathcal{T}$ we have assigned a certain family $\mathcal{A}(Z)$ of subsets of $Z$. Denote by $\mathcal{A}$ the collection of all these families. We say that $\mathcal{A}$ is closed under continuous substitutions if for each $X, Y \in \mathcal{T}$ and for every continuous function $f \in{ }^{X} Y$ we have $f^{-1}(A) \in \mathcal{A}(X)$ for every $A \in \mathcal{A}(Y)$. We shall call $\mathcal{A}$ a hereditary $\sigma$-class if $\mathcal{A}$ is closed under continuous substitutions and if for each $Z \in \mathcal{T}$ the following two conditions are satisfied:

(I) $\mathcal{A}(Z)$ is a $\sigma$-class;

(II) $\mathcal{A}(Z) \mid X=\mathcal{A}(X)$ for each closed $X \subseteq Z$.

Obviously, $\Sigma_{\alpha}^{0}, \alpha<\omega_{1}$, and $\Sigma_{n}^{1}, n \in \mathbb{N}$, are examples of hereditary $\sigma$-classes.

Let $X$ and $Y$ be any sets. For $A \subseteq X \times Y$ and $x \in X$ let $A_{x}=\{y \in Y$ : $(x, y) \in A\}$. If $\mathcal{A} \subseteq P(Y)$, a set $A \subseteq X \times Y$ is called a universal set for $\mathcal{A}$ if $\mathcal{A}=\left\{A_{x}: x \in X\right\}$. Recall that if $X$ and $Y$ are Polish spaces and $X$ is uncountable, then for any $\alpha<\omega_{1}$ there is a universal set for $\Sigma_{\alpha}^{0}(Y)$ in the class $\Sigma_{\alpha}^{0}(X \times Y)$, and the same is true for the classes $\Sigma_{n}^{1}, n \in \mathbb{N}$ (see [Mo]).

Let $F \in X \times Y \mathbb{R}$ and $(x, y) \in X \times Y$. We put $F_{x}(y)=F(x, y)$. A function $F \in X \times Y \mathbb{R}$ is called a universal function for a class $\mathcal{H} \subseteq Y \mathbb{R}$ if $\mathcal{H}=\left\{F_{x}: x \in X\right\}$.

We shall use the following known facts. Theorems 2.A and 2.B were formulated in [CMPS, Cor. 2.2 and Cor. 2.4] in a weaker form but, in fact, they are exactly the theorems proved there.

THEOREM 2.A. If $\mathcal{A}$ is a $\sigma$-class of subsets of $Z$ with the reduction property, then for every countable family of functions $\mathcal{H} \subseteq \mathbf{M} \mathcal{A}$ there exists $g \in \mathbf{M} \mathcal{A}$ such that inf $|f-g|>0$ for every $f \in \mathcal{H}$.

THEOREM 2.B. If $\mathcal{A}$ is a $\sigma$-class of subsets of $Z$ and $f \in \underline{\mathbf{M}} \mathcal{A}$, then the set $\{g \in \overline{\mathbf{M}} \mathcal{A}: \inf |f-g|>0\}$ is open and dense in $\overline{\mathbf{M}} \mathcal{A}$.

Theorem 2.C ([CM, Th. 2.1]). If $Z \in \mathcal{T}$ and $\mathcal{A}$ is a hereditary $\sigma$-class such that $\mathcal{A}(Z)$ has a universal set in $\mathcal{A}(\mathcal{C} \times Z)$, then there exists a universal function for $\underline{\mathbf{M}} \mathcal{A}(Z)$ in $\underline{\mathbf{M}} \mathcal{A}(\mathcal{C} \times Z)$.

Theorem 2.D (see, for instance, [CM, Prop. 1.1]). If $n \in \mathbb{N}$ and $\mathcal{A}$ is a $\sigma$-class then $f \in R \underline{\mathbf{M}}_{[-n, n]} \mathcal{A}$ if and only if there exists $f^{*} \in \underline{\mathbf{M}}_{[-n, n]} \mathcal{A}$ such that $f=f^{*} \mid \operatorname{dom} f$. A similar result holds for functions from $\overline{\mathbf{M}}_{[-n, n]} \mathcal{A}$. 
Now to formulate Theorem 2.E ([H, XIV, p. 277]) we introduce some notation used in $[\mathrm{H}]$.

A family $\mathcal{F}$ of real functions defined on a common domain $D$ will be called an ordinary function system if

(i) every real function which is constant on $D$ is in $\mathcal{F}$;

(ii) the maximum and minimum of two functions from $\mathcal{F}$ is in $\mathcal{F}$;

(iii) the sum, difference, product, and quotient (with nowhere vanishing denominator) of two functions from $\mathcal{F}$ is in $\mathcal{F}$.

An ordinary function system $\mathcal{F}$ is called complete if it also satisfies the following condition:

(iv) the limit of a uniformly convergent sequence of functions from $\mathcal{F}$ is in $\mathcal{F}$.

Let $\mathcal{A}$ and $\mathcal{B}$ be two families of functions. The function $f$ is said to be of class $(\mathcal{A}, \mathcal{B})$ if for each $c \in \mathbb{R}$ the set $f^{-1}((c, \infty))$ is in $\mathcal{A}$ and the set $f^{-1}([c, \infty))$ is in $\mathcal{B}([\mathrm{H}$, p. 267]).

Let $\mathcal{F}$ be a given family of functions defined on a common domain. Let $f$ range over $\mathcal{F}$, and let $g$ and $h$ range over all real functions which are pointwise limits of, respectively, nondecreasing and nonincreasing sequences of functions from $\mathcal{F}$. Then the sets of the form $f^{-1}([c, \infty)), g^{-1}((c, \infty))$, $h^{-1}([c, \infty))$ will be called N, P, Q sets, respectively ([H, p. 270]). Countable intersections of $\mathrm{N}$ sets will be called $\mathrm{N}_{\delta}$ sets. $\mathcal{P}$ and $\mathcal{Q}$ will stand for the families of all $\mathrm{P}$ and $\mathrm{Q}$ sets respectively.

The functions forming the least complete ordinary function system over $\mathcal{F}$ will be called v functions ([H, VII, p. 272]).

The following theorem was proved in [H, XIV, p. 277].

THEOREM 2.E. Let $\mathcal{F}$ be an ordinary function system. If $Q_{0}$ is a $\mathrm{Q}$ set, then each function $\phi: Q_{0} \rightarrow \mathbb{R}$ which is of class $\left(\mathcal{P}\left|Q_{0}, \mathcal{Q}\right| Q_{0}\right)$ can be extended to a function of class $(\mathcal{P}, \mathcal{Q})$, that is (see [H, VII, p. 272]), to a v function.

We now derive a corollary we shall use in the sequel.

COROLlary 2.F. If $\mathcal{A}$ is a $\sigma$-class of subsets of some set $Z$ and if $\phi \in$ $\mathbf{M}\left(\mathcal{A}^{\prime} \mid S\right)$ where $S \in \mathcal{A}_{\delta}$, then $\phi$ can be extended to some $\phi^{*} \in \mathbf{M} \mathcal{A}^{\prime}$.

Proof. Notice that all $B \in \mathcal{A}$ are $\mathrm{N}$ sets for the ordinary function system $\mathbf{M} \mathcal{A}^{\prime}$, because $\chi_{B} \in \mathbf{M} \mathcal{A}^{\prime}$. Thus the sets from $\mathcal{A}_{\delta}$ are $\mathrm{N}_{\delta}$ sets and therefore $\mathrm{Q}$ sets ([H, VI, p. 271]). Thus, by Theorem 2.E, the function $\phi$ can be extended to a $\mathrm{v}$ function $\phi^{*}$. But, as $\mathbf{M} \mathcal{A}^{\prime}$ is a complete ordinary function system ([H, III, p. 268]), every v function is in $\mathbf{M} \mathcal{A}^{\prime}$. 


\section{Algebras of measurable functions}

Lemma 3.1. Let $\mathcal{A}$ be a $\sigma$-class of subsets of $Z$. Let $h \in \overline{\mathbf{M}} \mathcal{A},|\operatorname{Rg} h|<\aleph_{0}$, $v \in \overline{\mathbf{M}} \mathcal{A}$. Then for each $\varepsilon>0$ there exists $g \in \underline{\mathbf{M}}_{[-1,1]} \mathcal{A}$ such that $\|g\|<\varepsilon$ and $\inf |g+h-v|>0$.

Proof. Let $\operatorname{Rg} h=\left\{\alpha_{1}, \ldots, \alpha_{n}\right\}, \alpha_{1}<\ldots<\alpha_{n}$. Let $A_{i}=\{z \in Z$ : $\left.h(z)=\alpha_{i}\right\}, i \leq n$. Assume $\varepsilon<1$. By Theorem 2.B for each $i \leq n$ there exists $g_{i} \in \underline{\mathbf{M}}_{[-1,1]}\left(\mathcal{A} \mid A_{i}\right)$ with inf $\left|g_{i}+\alpha_{i}-v\right|>0$ and $\left\|g_{i}-\varepsilon(n-i) /(2 n)\right\|<$ $\varepsilon /(4 n)$. Observe that $\sup g_{i+1}<\inf g_{i}, i=1, \ldots, n-1$. Define $g(z)=g_{i}(z)$ for $z \in A_{i}, i \leq n$. To see that $g \in \underline{\mathbf{M}}_{[-1,1]} \mathcal{A}$ we check that $g^{-1}((a, 1]) \in \mathcal{A}$ for each $a \in[0,1]$. Assume first that

$$
\min g_{k} \leq a \leq \max g_{k}
$$

for some $k \leq n$. Then

$$
g^{-1}((a, 1])=\bigcup_{i=1}^{k-1} A_{i} \cup g_{k}^{-1}((a, 1])=\bigcup_{i=1}^{k-1} A_{i} \cup\left(B \cap A_{k}\right)
$$

for some $B \in \mathcal{A}$. Further, we have

$$
\bigcup_{i=1}^{k-1} A_{i} \cup\left(B \cap A_{k}\right)=\left(\bigcup_{i=1}^{k-1} A_{i}\right) \cup\left(\left(\bigcup_{i=1}^{k} A_{i}\right) \cap B\right) \in \mathcal{A} .
$$

If (1) is not satisfied one can easily see that either

$$
g^{-1}((a, 1])=\bigcup_{i=1}^{k} A_{i} \in \mathcal{A}
$$

for some $k \leq n$, or $g^{-1}((a, 1])=\emptyset$.

We now apply Lemma 3.1 to prove the following:

Lemma 3.2. Let $w \in \overline{\mathbf{M}} \mathcal{A}$. Then the set $\left\{(l, u) \in \underline{\mathbf{M}}_{[-1,1]} \mathcal{A} \times \overline{\mathbf{M}}_{[-1,1]} \mathcal{A}\right.$ : $\inf |u+l-w|>0\}$ is residual in $\underline{\mathbf{M}}_{[-1,1]} \mathcal{A} \times \overline{\mathbf{M}}_{[-1,1]} \mathcal{A}$, in fact open and dense.

Proof. Let $u \in \overline{\mathbf{M}}_{[-1,1]} \mathcal{A}, l \in \underline{\mathbf{M}}_{[-1,1]} \mathcal{A}, \varepsilon>0$. Let $n \in \mathbb{N}$ and $n>\varepsilon^{-1}$. Define $A_{i}=u^{-1}\left(\left[(i-1) n^{-1}, i n^{-1}\right)\right)$ for $i \in\{-n+1, \ldots, n-1\}$, $A_{n}=u^{-1}\left(\left[(n-1) n^{-1}, 1\right]\right)$ and $h=\sum_{i=-n+1}^{n}(i-1) n^{-1} \chi_{A_{i}}$. Obviously, $h \in$ $\overline{\mathbf{M}}_{[-1,1]} \mathcal{A},\|u-h\|<\varepsilon$ and $|\operatorname{Rg} h|<\aleph_{0}$. Let $l^{\prime}=\max (\min (l, 1-\varepsilon),-1+\varepsilon)$ and $-l^{\prime}+w=v$. The functions $h$ and $v$ satisfy the conditions of the hypothesis of Lemma 3.1 and, by that lemma, there exists $g \in \mathbf{M}_{[-1,1]} \mathcal{A}$ such that $\|g\|<\varepsilon$ and $\delta=\inf \left|g+h+l^{\prime}-w\right|>0$. Of course $\left(l^{\prime}+g, h\right) \in$ $\underline{\mathbf{M}}_{[-1,1]} \mathcal{A} \times \overline{\mathbf{M}}_{[-1,1]} \mathcal{A}$ and for any pair $(\widetilde{l}, \widetilde{u}) \in \underline{\mathbf{M}}_{[-1,1]} \mathcal{A} \times \overline{\mathbf{M}}_{[-1,1]} \mathcal{A}$ such that $\|\widetilde{u}-h\|<\delta / 2$ and $\left\|\widetilde{l}-\left(l^{\prime}+g\right)\right\|<\delta / 2$ we have inf $|\widetilde{u}+\widetilde{l}-w|>0$. 
We also need the following dual lemma.

Lemma 3.3. Let $w \in \underline{\mathbf{M}} \mathcal{A}$. Then the set $\left\{(l, u) \in \underline{\mathbf{M}}_{[-1,1]} \mathcal{A} \times \overline{\mathbf{M}}_{[-1,1]} \mathcal{A}\right.$ : $\inf |u+l-w|>0\}$ is residual in $\underline{\mathbf{M}}_{[-1,1]} \mathcal{A} \times \overline{\mathbf{M}}_{[-1,1]} \mathcal{A}$, in fact open and dense.

From Lemmas 3.2 and 3.3 and the Baire category theorem we derive the following corollary.

COROllary 3.4. If $\mathcal{A}$ is a $\sigma$-class of subsets of $Z$, then for every countable family $\mathcal{H} \subseteq \underline{\mathbf{M}} \mathcal{A} \cup \overline{\mathbf{M}} \mathcal{A}$ there exists $f \in \underline{\mathbf{M}}_{[-1,1]} \mathcal{A}(Z)+\overline{\mathbf{M}}_{[-1,1]} \mathcal{A}(Z)$ such that $f(t) \neq g(t)$ for every $g \in \mathcal{H}$ and every $t \in Z$.

We are now able to prove our first decomposition theorem. The scheme of the proof is, in fact, the same as for Theorem 3.2 of [CMPS].

TheOREM 3.5. Let $\mathcal{A}$ be a hereditary $\sigma$-class, and let $Z \in \mathcal{T}$ be uncountable and such that $\mathcal{A}(Z)$ has a universal set in $\mathcal{A}(\mathcal{C} \times Z)$. Then there exists $f \in \underline{\mathbf{M}}_{[-1,1]} \mathcal{A}(Z)+\overline{\mathbf{M}}_{[-1,1]} \mathcal{A}(Z)$ such that there $i s$ no countable partition of $Z, Z=\bigcup\left\{Z_{n}: n \in \mathbb{N}\right\}$, such that $f \mid Z_{n} \in \underline{\mathbf{M}}\left(\mathcal{A}(Z) \mid Z_{n}\right) \cup \overline{\mathbf{M}}\left(\mathcal{A}(Z) \mid Z_{n}\right)$ for every $n \in \mathbb{N}$. In other words,

$$
\operatorname{dec}\left(\underline{\mathbf{M}}_{[-1,1]} \mathcal{A}(Z)+\overline{\mathbf{M}}_{[-1,1]} \mathcal{A}(Z), R \underline{\mathbf{M}} \mathcal{A}(Z) \cup R \overline{\mathbf{M}} \mathcal{A}(Z)\right)>\aleph_{0} .
$$

Proof. Let $C \subseteq Z$ be homeomorphic to $\mathcal{C}$. Let $F \in \underline{\mathbf{M}}_{[-1,1]} \mathcal{A}(C \times$ $Z$ ) and $G \in \overline{\mathbf{M}}_{[-1,1]} \mathcal{A}(C \times Z)$ be universal functions for $\underline{\mathbf{M}}_{[-1,1]} \mathcal{A}(Z)$ and $\overline{\mathbf{M}}_{[-1,1]} \mathcal{A}(Z)$, respectively. Let $\pi=\left(\pi_{1}, \pi_{2}, \ldots\right): C \rightarrow{ }^{\mathbb{N}} C$ be a fixed homeomorphism. For every $n \in \mathbb{N}$ let $f_{n} \in \underline{\mathbf{M}}_{[-1,1]} \mathcal{A}(Z)$ and $g_{n} \in \overline{\mathbf{M}}_{[-1,1]} \mathcal{A}(Z)$ be such that $f_{n}(t)=F\left(\pi_{n}(t), t\right)$ and $g_{n}(t)=G\left(\pi_{n}(t), t\right)$ for every $t \in C$. By Corollary 3.4 there exists $f \in \underline{\mathbf{M}}_{[-1,1]} \mathcal{A}(Z)+\overline{\mathbf{M}}_{[-1,1]} \mathcal{A}(Z)$ such that $f(t) \neq g_{n}(t)$ and $f(t) \neq f_{n}(t)$ for each $t \in Z$.

Now assume that $f=\bigcup\left\{h_{k}: k \in \mathbb{N}\right\}$ and $h_{k} \in R \underline{\mathbf{M}} \mathcal{A}(Z) \cup R \overline{\mathbf{M}} \mathcal{A}(Z)$ for each $k \in \mathbb{N}$. Let $h_{k}^{*} \in \underline{\mathbf{M}} \mathcal{A}(Z) \cup \overline{\mathbf{M}} \mathcal{A}(Z)$ be an extension of $h_{k}$ (see Theorem 2.D). There exists $c \in C$ such that for every $k \in \mathbb{N}$ and for every $t \in Z$ either $h_{k}^{*}(t)=F\left(\pi_{k}(c), t\right)$ or $h_{k}^{*}(t)=G\left(\pi_{k}(c), t\right)$. Thus $f(c) \in\left\{f_{k}(c)\right.$ : $k \in \mathbb{N}\} \cup\left\{g_{k}(c): k \in \mathbb{N}\right\}$, which is impossible.

Corollary 3.6. If $Z$ is an uncountable Polish space, then for any $\alpha<\omega_{1}$

$$
\operatorname{dec}\left(\mathbf{L}_{\alpha}(Z)+\mathbf{U}_{\alpha}(Z), R \mathbf{L}_{\alpha}(Z) \cup R \mathbf{U}_{\alpha}(Z)\right)>\aleph_{0} .
$$

Corollary 3.7. If $Z$ is an uncountable Polish space, then for any $n \in \mathbb{N}$

$$
\operatorname{dec}\left(\underline{\mathbf{M}} \Sigma_{n}^{1}(Z)+\overline{\mathbf{M}} \Sigma_{n}^{1}(Z), R \underline{\mathbf{M}} \Sigma_{n}^{1}(Z) \cup R \overline{\mathbf{M}} \Sigma_{n}^{1}(Z)\right)>\aleph_{0} .
$$

We shall need the following lemma. 
LEMma 3.8. If $\mathcal{A}$ is a hereditary $\sigma$-class, $Z \in \mathcal{T}$ and $\mathcal{A}(Z)$ has a universal set in $\mathcal{A}(\mathcal{C} \times Z)$, then for every $n \in \mathbb{N}$ the class $\underline{\mathbf{M}}_{[-n, n]} \mathcal{A}(Z)+\overline{\mathbf{M}}_{[-n, n]} \mathcal{A}(Z)$ has a universal function in $\underline{\mathbf{M}}_{[-n, n]} \mathcal{A}(\mathcal{C} \times Z)+\overline{\mathbf{M}}_{[-n, n]} \mathcal{A}(\mathcal{C} \times Z)$.

Proof. Let $\phi=\left(\phi_{1}, \phi_{2}\right): \mathcal{C} \rightarrow \mathcal{C}^{2}$ be any homeomorphism. Let $F \in$ $\underline{\mathbf{M}}_{[-n, n]} \mathcal{A}(\mathcal{C} \times Z)$ and $G \in \overline{\mathbf{M}}_{[-n, n]} \mathcal{A}(\mathcal{C} \times Z)$ be universal functions for $\underline{\mathbf{M}}_{[-n, n]} \mathcal{A}(Z)$ and $\underline{\mathbf{M}}_{[-n, n]} \mathcal{A}(Z)$, respectively. Then $H(c, x)=F\left(\phi_{1}(c), x\right)+$ $G\left(\phi_{2}(c), x\right)$ is a universal function for $\underline{\mathbf{M}}_{[-n, n]} \mathcal{A}(Z)+\overline{\mathbf{M}}_{[-n, n]} \mathcal{A}(Z)$.

Lemma 3.9. Let $\mathcal{A}$ be a $\sigma$-class of subsets of some set $Z$. If $A \in r(\mathcal{A})$ then $\chi_{A} \in \mathrm{b} \underline{\mathbf{M}} \mathcal{A}+\mathrm{b} \overline{\mathbf{M}} \mathcal{A}$.

Proof. The family

$$
\mathcal{S}=\left\{A \in r(\mathcal{A}): \chi_{A} \in \mathrm{b} \underline{\mathbf{M}} \mathcal{A}+\mathrm{b} \overline{\mathbf{M}} \mathcal{A}\right\}
$$

is obviously closed under finite intersections and taking complements and at the same time $\mathcal{A} \subseteq \mathcal{S}$. Thus $\mathcal{S}=r(\mathcal{A})$.

Lemma 3.10. Let $n \in \mathbb{N}$, let $\mathcal{A}$ be a $\sigma$-class of subsets of some set $Z$ and $g \in \underline{\mathbf{M}}_{[-N, N]} \mathcal{A}+\overline{\mathbf{M}}_{[-N, N]} \mathcal{A}, N \in \mathbb{N}$. Then there exists $w \in \mathrm{b} \underline{\mathbf{M}} \mathcal{A}+\mathrm{b} \overline{\mathbf{M}} \mathcal{A}$ such that $\|g-w\|<2^{-n+1}$ and

$$
w=\sum_{i=-2^{n+1} N}^{2^{n+1} N} i \cdot 2^{-n} \chi_{A_{i}},
$$

where the sets $A_{i}$ are pairwise disjoint and, for each $i, A_{i} \in r(\mathcal{A})$ and therefore $\chi_{A_{i}} \in \mathrm{b} \underline{\mathbf{M}} \mathcal{A}+\mathrm{b} \overline{\mathbf{M}} \mathcal{A}$.

Proof. Let $g=u+l, l \in \underline{\mathbf{M}}_{[-N, N]} \mathcal{A}$ and $u \in \overline{\mathbf{M}}_{[-N, N]} \mathcal{A}$. Let $B_{i}=$ $l^{-1}\left(\left(i \cdot 2^{-n},(i+1) \cdot 2^{-n}\right]\right)$ and $C_{i}=u^{-1}\left(\left[i \cdot 2^{-n},(i+1) \cdot 2^{-n}\right)\right)$. The sets $B_{i}$ and $C_{i}$ belong to $r(\mathcal{A})$. Let

$$
w=\sum_{i=-2^{n} N}^{2^{n} N} i \cdot 2^{-n} \chi_{B_{i-1}}+\sum_{i=-2^{n} N}^{2^{n} N} i \cdot 2^{-n} \chi_{C_{i}}=\sum_{j=-2^{n+1} N}^{2^{n+1} N} j \cdot 2^{-n} \chi_{A_{j}},
$$

where $A_{j}=\bigcup\left\{B_{i} \cap C_{k}: i+k+1=j\right\}$. It follows from Lemma 3.9 that $w$ is the function we need.

Lemma 3.11. Let $\mathcal{A}$ be a $\sigma$-class of subsets of some set $Z$. Let $f, g \in$ $\mathrm{b} \underline{\mathbf{M}} \mathcal{A}+\mathrm{b} \overline{\mathbf{M}} \mathcal{A}$. Let $\varepsilon>0$. Then there exists $h \in \mathrm{b} \underline{\mathbf{M}} \mathcal{A}+\mathrm{b} \overline{\mathbf{M}} \mathcal{A}$ such that $\|h-g\|<3 \varepsilon$ and $\inf |h-f| \geq \varepsilon / 3$.

Pr o o f. By Lemma 3.10 there exist $\phi=\sum_{i=1}^{N} c_{i} \chi_{A_{i}}$ and $\psi=\sum_{j=1}^{M} d_{j} \chi_{B_{j}}$ such that $A_{i}, B_{j} \in r(\mathcal{A}), i \leq N, j \leq M$, the sets $A_{i}$ are pairwise disjoint, the $B_{j}$ are pairwise disjoint, $\|f-\phi\|<\varepsilon / 3$, and $\|g-\psi\|<\varepsilon / 3$. Taking appropriate intersections we can assume that for each $j \leq M$ there exists 
$i \leq N$ such that $B_{j} \subseteq A_{i}$. Let $B_{j} \subseteq A_{i}$. Then we define $h$ on $B_{j}$ in the following way:

$$
h \mid B_{j}= \begin{cases}\psi \mid B_{j} & \text { if }\left|d_{j}-c_{i}\right| \geq 2 \varepsilon / 3 \\ \psi \mid B_{j}+2 \varepsilon & \text { if }\left|d_{j}-c_{i}\right|<2 \varepsilon / 3\end{cases}
$$

Lemma 3.12. If $\mathcal{A}$ is a $\sigma$-class of subsets of $Z$ then for every countable family $\mathcal{G} \subseteq \operatorname{cl}(\mathrm{b} \underline{\mathbf{M}} \mathcal{A}+\mathrm{b} \overline{\mathbf{M}} \mathcal{A})$ there exists $g \in \operatorname{cl}(\mathrm{b} \underline{\mathbf{M}} \mathcal{A}+\mathrm{b} \overline{\mathbf{M}} \mathcal{A})$ such that $\inf |f-g|>0$ for every $f \in \mathcal{G}$.

P r o o f. By Lemma 3.11 for any $f \in \mathcal{G}$ the family $\{h \in \operatorname{cl}(\mathrm{b} \underline{\mathbf{M}} \mathcal{A}+\mathrm{b} \overline{\mathbf{M}} \mathcal{A})$ : $\inf |f-h|>0\}$ is residual in $\operatorname{cl}(\mathrm{b} \mathbf{M} \mathcal{A}+\mathrm{b} \overline{\mathbf{M}} \mathcal{A})$. As the latter space is complete, the lemma follows by the Baire category theorem.

Theorem 3.13. Let $\mathcal{A}$ be a hereditary $\sigma$-class on $\mathcal{T}$. Let $\mathcal{A}(Z)$, for some uncountable $Z \in \mathcal{T}$, have a universal set in $\mathcal{A}(\mathcal{C} \times Z)$. Then there exists a function $f \in \operatorname{cl}(\mathrm{b} \mathbf{M} \mathcal{A}(Z)+\mathrm{b} \overline{\mathbf{M}} \mathcal{A}(Z))$ for which there is no countable partition $Z=\bigcup\left\{Z_{m}: m \in \mathbb{N}\right\}$ such that $f\left|Z_{m} \in \underline{\mathbf{M}} \mathcal{A}(Z)\right| Z_{m}+\overline{\mathbf{M}} \mathcal{A}(Z) \mid Z_{m}$ for each $m \in \mathbb{N}$. In other words,

$$
\operatorname{dec}(\operatorname{cl}(\mathrm{b} \underline{\mathbf{M}} \mathcal{A}(Z)+\mathrm{b} \overline{\mathbf{M}} \mathcal{A}(Z)), R(\underline{\mathbf{M}} \mathcal{A}(Z)+\overline{\mathbf{M}} \mathcal{A}(Z)))>\aleph_{0} .
$$

Pr o of. Let $C \subseteq Z$ be homeomorphic to $\mathcal{C}$. By Lemma 3.8 for each $n \in \mathbb{N}$ there exists $G_{n} \in \underline{\mathbf{M}}_{[-n, n]} \mathcal{A}(C \times Z)+\overline{\mathbf{M}}_{[-n, n]} \mathcal{A}(C \times Z)$ which is a universal function for $\underline{\mathbf{M}}_{[-n, n]} \mathcal{A}(Z)+\overline{\mathbf{M}}_{[-n, n]} \mathcal{A}(Z)$. Let $\pi=\left(\pi_{1}, \pi_{2}, \ldots\right): C \rightarrow{ }^{\mathbb{N}} C$ be a fixed homeomorphism. Let $g_{n}(t)=G_{n}\left(\pi_{n}(t), t\right)$ for every $t \in C$. It is easy to see that $g_{n} \in \underline{\mathbf{M}}_{[-n, n]} \mathcal{A}(Z)+\overline{\mathbf{M}}_{[-n, n]} \mathcal{A}(Z)$. By Lemma 3.12 there exists $f \in \operatorname{cl}(\mathrm{b} \underline{\mathbf{M}} \mathcal{A}(Z)+\mathrm{b} \overline{\mathbf{M}} \mathcal{A}(Z))$ such that $f(t) \neq g_{n}(t)$ for each $t \in Z$ and for each $n \in \mathbb{N}$.

Assume there is a partition $Z=\bigcup\left\{Z_{m}: m \in \mathbb{N}\right\}$ such that $f \mid Z_{m} \in$ $\underline{\mathbf{M}}\left(\mathcal{A}(Z) \mid Z_{m}\right)+\overline{\mathbf{M}}\left(\mathcal{A}(Z) \mid Z_{m}\right)$ for each $m \in \mathbb{N}$. Let $f \mid Z_{m}=l_{m}+u_{m}$ where $l_{m} \in \underline{\mathbf{M}} \mathcal{A}(Z) \mid Z_{m}$ and $u_{m} \in \overline{\mathbf{M}} \mathcal{A}(Z) \mid Z_{m}$. Let $Z_{m, n}=\left\{x \in Z_{m}\right.$ : $\left|l_{m}(x)\right| \leq n$ and $\left.\left|u_{m}(x)\right| \leq n\right\}$. Of course $\bigcup\left\{Z_{m, n}: n \in \mathbb{N}\right\}=Z_{m}$. Let $l_{m, n} \in \underline{\mathbf{M}}_{[-n, n]} \mathcal{A}(Z)$ and $u_{m, n} \in \overline{\mathbf{M}}_{[-n, n]} \mathcal{A}(Z)$ be extensions of $l_{m} \mid Z_{m, n}$ and $u_{m} \mid Z_{m, n}$, respectively (see Theorem 2.D). There exists $c \in C$ such that for each pair $m, n \in \mathbb{N}$ there exists $i(m, n) \in \mathbb{N}$ such that $l_{m, n}(t)+u_{m, n}(t)=$ $G_{i(m, n)}\left(\pi_{i(m, n)}(c), t\right)$ for each $t \in \mathbb{N}$. Let $c \in Z_{m, n}$ for some $m, n \in \mathbb{N}$. Then $f(c)=l_{m, n}(c)+u_{m, n}(c)=g_{i(m, n)}(c)$, which is a contradiction.

Corollary 3.14. If $Z$ is an uncountable Polish space, then for any $\alpha<\omega_{1}$

$$
\operatorname{dec}\left(\operatorname{cl}\left(\mathrm{b} \mathbf{L}_{\alpha}(Z)+\mathrm{b} \mathbf{U}_{\alpha}(Z)\right), R\left(\mathbf{L}_{\alpha}(Z)+\mathbf{U}_{\alpha}(Z)\right)\right)>\aleph_{0} .
$$

Corollary 3.15. If $Z$ is an uncountable Polish space, then for any $n \in \mathbb{N}$

$$
\operatorname{dec}\left(\operatorname{cl}\left(\mathrm{b} \underline{\mathbf{M}} \Sigma_{n}^{1}(Z)+\mathrm{b} \overline{\mathbf{M}} \Sigma_{n}^{1}(Z)\right), R\left(\underline{\mathbf{M}} \Sigma_{n}^{1}(Z)+\overline{\mathbf{M}} \Sigma_{n}^{1}(Z)\right)\right)>\aleph_{0} .
$$


LEMMA 3.16. Let $\mathcal{A}$ be a $\sigma$-class of subsets of some set $Z$. If $f \in \operatorname{cl}(\mathrm{bM} \mathcal{A}$ $+\mathrm{b} \overline{\mathbf{M}} \mathcal{A})$, then for given $n \in \mathbb{N}$ and $\delta>0$ there exists $v \in \mathrm{b} \underline{\mathbf{M}} \mathcal{A}+\mathrm{b} \overline{\overline{\mathbf{M}}} \mathcal{A}$ such that $\|f-v\|<2^{-n+1}, \operatorname{Rg} v \subseteq\left\{i \cdot 2^{-n}: i \in \mathbb{Z}\right\}$ and $v(x)<v(y)$ implies $f(x)<f(y)+\delta$ for all $x, y \in Z$.

Proof. Let $m \in \mathbb{N}, m>n+1$ and $2^{-m+2}<\delta$. Let $g \in \mathrm{b} \underline{\mathbf{M}} \mathcal{A}+\mathrm{b} \overline{\mathbf{M}} \mathcal{A}$ and $\|f-g\|<2^{-m}$. By Lemma 3.10 there exists

$$
w=\sum_{i=-M}^{M} i \cdot 2^{-m-1} \chi_{A_{i}}
$$

where $M \in \mathbb{N},\|g-w\|<2^{-m}$, the sets $A_{i}$ are pairwise disjoint and, for each $i, A_{i} \in r(\mathcal{A})$ and thus $\chi_{A_{i}} \in \mathrm{b} \underline{\mathbf{M}} \mathcal{A}+\mathrm{b} \overline{\mathbf{M}} \mathcal{A}$. Then $\|w-f\|<2^{-m+1}$. Let $v(x)=\left[2^{n} w(x)\right] \cdot 2^{-n}$. Obviously $\operatorname{Rg} v \subseteq\left\{i \cdot 2^{-n}: i \in \mathbb{Z}\right\}$ and $\|f-v\|<2^{-n+1}$. If $v(x)<v(y)$ then $w(x)<w(y)$, whence $f(x)<f(y)+2^{-m+2}<f(y)+\delta$.

THEOREM 3.17. Let $\mathcal{A}$ be a $\sigma$-class of subsets of some set $Z$. Then each $f \in \operatorname{cl}(\mathrm{b} \underline{\mathbf{M}} \mathcal{A}+\mathrm{b} \overline{\mathbf{M}} \mathcal{A})$ can be expressed as the superposition $f=g \circ h$ where $h \in \mathrm{b} \underline{\mathbf{M}}_{[-1,1]} \mathcal{A}+\mathrm{b} \overline{\mathbf{M}}_{[-1,1]} \mathcal{A}$ and $g \in C([-2,2]) \cap V B([-2,2])$.

Proof. Let $f \in \operatorname{cl}(\mathrm{bM} \mathcal{A}+\mathrm{b} \overline{\mathbf{M}} \mathcal{A})$. Let $\|f\|<N \in \mathbb{N}$. The function $f$ is the uniform limit $f=\lim _{n \rightarrow \infty} v_{n}$ of functions $v_{n} \in \mathrm{b} \underline{\mathbf{M}} \mathcal{A}+\mathrm{b} \overline{\mathbf{M}} \mathcal{A}$. By Lemma 3.16 we can assume that $\operatorname{Rg} v_{n} \subseteq\left\{i / 2^{n}: i \in \mathbb{Z}\right\},\left\|f-v_{n}\right\|<2^{-n+1}$ and that

$$
v_{n}(x)<v_{n}(y) \text { implies } f(x)<f(y)+\delta_{n},
$$

where $\delta_{n}=(N+1)^{-1} \cdot 2^{-2 n-2}$. Let $v_{n}=l_{n}+u_{n}$, where $l_{n} \in \mathrm{b} \underline{\mathbf{M}} \mathcal{A}$ and $u_{n} \in \mathrm{b} \overline{\mathbf{M}} \mathcal{A}$. Let $\mathbf{v}(x)=\left(v_{1}(x), v_{2}(x), \ldots\right)$. Let $s$ be the function defined on $\operatorname{Rg} \mathbf{v}$ as $s\left(v_{1}(x), v_{2}(x), \ldots\right)=\lim _{n \rightarrow \infty} v_{n}(x)$. Of course $f=s \circ \mathbf{v}$.

Now, let $\alpha_{n}, n \in \mathbb{N}$, satisfy the following conditions:

$$
\begin{aligned}
& 1^{\mathrm{o}} \alpha_{n}>0, n \in \mathbb{N} ; \\
& 2^{\mathrm{o}} \alpha_{n} \sup \left|l_{n}\right|<2^{-n} \text { and } \alpha_{n} \sup \left|u_{n}\right|<2^{-n} ; \\
& 3^{\mathrm{o}} \alpha_{n} 2^{-n}>2 \sum_{i>n} \alpha_{i}\left(\sup \left|l_{i}\right|+\sup \left|u_{i}\right|\right) .
\end{aligned}
$$

Let

$$
\phi(\mathbf{v}(x))=\sum_{n=1}^{\infty} \alpha_{n} v_{n}(x)=\sum_{n=1}^{\infty} \alpha_{n} l_{n}(x)+\sum_{n=1}^{\infty} \alpha_{n} u_{n}(x) .
$$

The convergence of the series follows from $2^{\circ}$. From $3^{\circ}$ it follows that $\phi$ is $1-1$. We have $f=s \circ \mathbf{v}=\left(s \circ \phi^{-1}\right) \circ(\phi \circ \mathbf{v})$. Of course $\phi \circ \mathbf{v} \in \underline{\mathbf{M}}_{[-1,1]} \mathcal{A}+\overline{\mathbf{M}}_{[-1,1]} \mathcal{A}$ and we put $h=\phi \circ \mathbf{v}$.

Now we show that $\varphi=s \circ \phi^{-1}$ can be extended to a function $g \in$ $V B([-2,2]) \cap C([-2,2])$. To this end it is enough to show that $\varphi$ can be extended to a continuous function $\widetilde{\varphi}$ on $\operatorname{cl}(\operatorname{dom} \varphi)$ and that $\varphi$ is of bounded variation on its domain. 
Let $s_{k}=\sum_{n=1}^{\infty} \alpha_{n} v_{n}\left(x_{k}\right)$ and $t_{k}=\sum_{n=1}^{\infty} \alpha_{n} v_{n}\left(x_{k}^{\prime}\right)$ and $s_{k} \nearrow q \swarrow t_{k}$. We shall show that the sequences $\varphi\left(s_{k}\right)$ and $\varphi\left(t_{k}\right)$ converge to the same limit $\widetilde{\varphi}(q)$. For each $n \in \mathbb{N}$ there is some $k(n)$ such that $v_{n}\left(x_{k}\right)=v_{n}\left(x_{k}^{\prime}\right)$ for $k>$ $k(n)$. Indeed, as $\phi$ preserves the lexicographic order on $\mathbf{v}(Z)$ the sequence $v_{1}\left(x_{k}\right)$ is nondecreasing and, as $\left|\operatorname{Rg} v_{1}\right|<\aleph_{0}$, it is constant for $k \geq n_{1}$, for some $n_{1} \in \mathbb{N}$. Then for $k \geq n_{1}$ the sequence $v_{2}\left(x_{k}\right)$ is nondecreasing and is constant for $k \geq n_{2}$ for some $n_{2} \geq n_{1}$. Inductively we prove that for each $m \in \mathbb{N}$ the sequence $v_{m}\left(x_{k}\right)$ is constant for $k \geq n_{m}$ for some $n_{m} \geq n_{m-1}$. Similarly, putting $n_{0}^{\prime}=0$, by induction we show that for each $m \in \mathbb{N}$ the sequence $v_{m}\left(x_{k}^{\prime}\right)$ is nonincreasing for $k \geq n_{m-1}^{\prime}$ and constant for $k \geq n_{m}^{\prime}$ for some $n_{m}^{\prime} \geq n_{m-1}^{\prime}$.

If $v_{1}\left(x_{n_{1}}\right)<v_{1}\left(x_{n_{1}^{\prime}}^{\prime}\right)$ then for all $k \geq \max \left(n_{1}, n_{1}^{\prime}\right)$ we would have $v_{1}\left(x_{k}^{\prime}\right)=$ $v_{1}\left(x_{n_{1}^{\prime}}^{\prime}\right)>v_{1}\left(x_{n_{1}}\right)=v_{1}\left(x_{k}\right)$ and by $3^{\circ}, t_{k}-s_{k}>\varepsilon$ for some fixed $\varepsilon>0$, which is a contradiction. Inductively $v_{m}\left(x_{n_{m}}\right)=v_{m}\left(x_{n_{m}^{\prime}}^{\prime}\right)$ for each $m \in \mathbb{N}$. As the sequence $v_{m}$ is uniformly convergent to $f$ and for any $m \in \mathbb{N}$ we have $v_{m}\left(x_{k}\right)=v_{m}\left(x_{k}^{\prime}\right)$ for $k \geq \max \left(n_{m}, n_{m}^{\prime}\right)$, the sequences $f\left(x_{k}\right)=\varphi\left(s_{k}\right)$ and $f\left(x_{k}^{\prime}\right)=\varphi\left(t_{k}\right)$ are convergent and $f\left(x_{k}\right)-f\left(x_{k}^{\prime}\right) \rightarrow 0$. Thus $\varphi$ can be extended to a continuous function $\widetilde{\varphi}$ defined on $\operatorname{cl}(\operatorname{dom} \varphi)$.

Now we show that $\varphi$ is of bounded variation on its domain. Let $t_{1}<\ldots$ $<t_{m}$, where $t_{i}=\sum_{n=1}^{\infty} \alpha_{n} v_{n}\left(x_{i}\right)=\phi \circ \mathbf{v}\left(x_{i}\right), i \leq m$. We shall estimate the sum $\sum_{i \in \mathcal{I}}\left(\varphi\left(t_{i}\right)-\varphi\left(t_{i+1}\right)\right)$, where $\mathcal{I} \subseteq\{1, \ldots, m-1\}$ is the set of all $i$ for which $\varphi\left(t_{i}\right)-\varphi\left(t_{i+1}\right)>0$. Let $A_{n}=\left\{i \in \mathcal{I}: \min \left\{j: v_{j}\left(x_{i}\right)<v_{j}\left(x_{i+1}\right)\right\}\right.$ $=n\}$. We have

$$
\sum_{i \in \mathcal{I}}\left(\varphi\left(t_{i}\right)-\varphi\left(t_{i+1}\right)\right)=\sum_{n=1}^{\infty} \sum_{i \in A_{n} \cap \mathcal{I}}\left(\varphi\left(t_{i}\right)-\varphi\left(t_{i+1}\right)\right) .
$$

For $i \in A_{n}$ we have, by $(*), \varphi\left(t_{i}\right)-\varphi\left(t_{i+1}\right)=f\left(x_{i}\right)-f\left(x_{i+1}\right)<\delta_{n}$, whence

$$
\sum_{i \in \mathcal{I}}\left(\varphi\left(t_{i}\right)-\varphi\left(t_{i+1}\right)\right)<\sum_{n=1}^{\infty} 2(N+1) \cdot 2^{n} \delta_{n}<1 .
$$

Thus $\varphi$ is of bounded variation.

By [Ma, Th. 2] we have the following converse theorem:

THEOREM 3.18. If $\mathcal{R}$ is any algebra of functions such that $\mathrm{cl} \mathcal{R}=\mathcal{R}$ then for any $f \in \mathrm{b} \mathcal{R}$ and any function $g$ continuous on a closed interval containing $\operatorname{Rg} f$ we have $g \circ f \in \mathcal{R}$.

Remark. Corollary 3.14 and Theorem 3.17 show that Theorem 14 in $[\mathrm{L}]$ is false. That there was a mistake in its proof in $[\mathrm{L}]$ was already noticed by A. Lindenbaum himself in [L, corr.].

Lemma 3.19. Let $\mathcal{A}$ be a $\sigma$-class of subsets of some set $Z$. Let $X \subseteq Z$. Then 
every $f \in \operatorname{cl}(\mathrm{b} \underline{\mathbf{M}}(\mathcal{A}(Z) \mid X)+\mathrm{b} \overline{\mathbf{M}}(\mathcal{A}(Z) \mid X))$ can be extended to a function $f^{*} \in \operatorname{cl}(\mathrm{b} \underline{\mathbf{M}} \mathcal{A}(Z)+\mathrm{b} \overline{\mathbf{M}} \mathcal{A}(Z))$.

Proof. By Theorem 3.17, $f=h \circ g$ where $h \in C(\mathbb{R})$ and $g \in$ $\mathrm{b} \underline{\mathbf{M}}(\mathcal{A}(Z) \mid X)+\mathrm{b} \overline{\mathbf{M}}(\mathcal{A}(Z) \mid X)$. The function $g$ can be extended to some $g^{*} \in \mathrm{b} \underline{\mathbf{M}} \mathcal{A}(Z)+\mathrm{b} \overline{\mathbf{M}} \mathcal{A}(Z)$. Then $f^{*}=h \circ g^{*} \in \operatorname{cl}(\mathrm{bM} \mathcal{A}(Z)+\mathrm{b} \overline{\mathbf{M}} \mathcal{A}(Z))$ by Theorem 3.18.

THEOREM 3.20. Let $\mathcal{A}$ be a hereditary $\sigma$-class on $\mathcal{T}$ and suppose $\Sigma_{1}^{0}(X) \subseteq$ $\mathcal{A}(X)$ for every $X \in \mathcal{T}$. Let $Z \in \mathcal{T}$ and suppose $\mathcal{A}(Z)$ has a universal set in $\mathcal{A}(\mathcal{C} \times Z)$. Then there is an $F \in \mathbf{M} \mathcal{A}^{\prime}(\mathcal{N} \times Z)$ which is a universal function for $\operatorname{cl}(\mathrm{b} \underline{\mathbf{M}} \mathcal{A}(Z)+\mathrm{b} \overline{\mathbf{M}} \mathcal{A}(Z))$.

Proof. By Lemma 3.8 there is a function $H \in \underline{\mathbf{M}}_{[-1,1]} \mathcal{A}(\mathcal{C} \times Z)+$ $\overline{\mathbf{M}}_{[-1,1]} \mathcal{A}(\mathcal{C} \times Z)$ universal for $\underline{\mathbf{M}}_{[-1,1]} \mathcal{A}(Z)+\overline{\mathbf{M}}_{[-1,1]} \mathcal{A}(Z)$. Let $\phi: \mathcal{N} \rightarrow \mathcal{C}$ be a continuous surjection $([\mathrm{Ku}, 37, \mathrm{I}$, Th. 1]). Let $G(w, x)=H(\phi(w), x)$ for $w \in \mathcal{N}$ and $x \in Z$. Then $G \in \mathbf{M} \mathcal{A}^{\prime}(\mathcal{N} \times Z)$ because $H \in \mathbf{M} \mathcal{A}^{\prime}(\mathcal{C} \times Z)$ and, as is easy to see, $\mathcal{A}^{\prime}$ is closed under continuous substitutions. Let $\psi: \mathcal{N} \rightarrow C([-2,2])$ be a continuous surjection ([Ku, 37, I, Th. 1]). We write $\psi_{w}(\cdot)$ for $\psi(w)$ in the sequel. Let $\xi=\left(\xi_{1}, \xi_{2}\right)$ be any homeomorphism from $\mathcal{N}$ onto $\mathcal{N}^{2}$. Let $F(w, x)=\psi_{\xi_{1}(w)}\left(G\left(\xi_{2}(w), x\right)\right)$. By Theorems 3.17 and 3.18, $F$ is universal for $\operatorname{cl}(\mathrm{b} \underline{\mathbf{M}} \mathcal{A}(Z)+\mathrm{b} \overline{\mathbf{M}} \mathcal{A}(Z))$.

We show that $F \in \mathbf{M} \mathcal{A}^{\prime}(\mathcal{N} \times Z)$. Let $\Psi(w, s)=\psi_{\xi_{1}(w)}(s), w \in \mathcal{N}$, $s \in[-2,2], \widetilde{G}(w, x)=G\left(\xi_{2}(w), x\right)$ and $\Phi(w, x)=(w, \widetilde{G}(w, x))$. We have $F(w, x)=\Psi(\Phi(w, x))$. Then $\widetilde{G} \in \mathbf{M} \mathcal{A}^{\prime}(\mathcal{N} \times Z)$ because $\mathcal{A}^{\prime}$ is closed under continuous substitutions. An easy argument shows that $\Psi$ is continuous. Finally, $\Phi^{-1}(U) \in \mathcal{A}^{\prime}(\mathcal{N} \times Z)$ for any open set $U \subseteq \mathcal{N} \times \mathbb{R}$ : indeed, as $U=\bigcup_{i=1}^{\infty} V_{i} \times W_{i}$, where the $V_{i}$ are open in $\mathcal{N}$ and $W_{i}$ are open in $\mathbb{R}$, we have

$$
\Phi^{-1}(U)=\bigcup_{i=1}^{\infty}\left(V_{i} \times Z\right) \cap \widetilde{G}^{-1}\left(W_{i}\right) \in \mathcal{A}^{\prime}(\mathcal{N} \times Z) .
$$

In the next theorem we add new assumptions on the hereditary $\sigma$-class $\mathcal{A}$ and the family $\mathcal{T}$. Namely, we assume that $\mathcal{T}$ satisfies the following stronger form of (i):

(i*) if $X \subseteq Z \in \mathcal{T}$ and $X$, as a subspace of $Z$, is completely metrizable by some metric $\rho$, then $(X, \rho) \in \mathcal{T}$.

We then assume that $\mathcal{A}$ satisfies for any $Z \in \mathcal{T}$ and $X \subseteq Z$ :

$\left(\mathrm{II}^{*}\right) \mathcal{A}(Z) \mid X=\mathcal{A}(X)$ where $X$ is considered with any metric $\rho$ such that $(X, \rho) \in \mathcal{T}$ is topologically a subspace of $Z$.

Assume also $\Sigma_{1}^{0} \subseteq \mathcal{A}$. 
However, the conditions imposed on $\mathcal{A}$ are not very restrictive as the classes $\Sigma_{\alpha}^{0}, \Sigma_{n}^{0}$ still satisfy them.

THEOREM 3.21. Let $\mathcal{A}$ be a hereditary $\sigma$-class satisfying $\left(\mathrm{II}^{*}\right)$. Let $Z \in \mathcal{T}$ be uncountable and suppose $\mathcal{A}(Z)$ has a universal set in $\mathcal{A}(\mathcal{C} \times Z)$. Then there exists $g \in \mathbf{M} \mathcal{A}^{\prime}(Z)$ for which there is no countable partition $Z=$ $\bigcup\left\{Z_{n}: n \in \mathbb{N}\right\}$ such that $g \mid Z_{n} \in \operatorname{cl}\left(\mathrm{bM}\left(\mathcal{A}(Z) \mid Z_{n}\right)+\mathrm{b} \overline{\mathbf{M}}\left(\mathcal{A}(Z) \mid Z_{n}\right)\right)$ for each $n \in \mathbb{N}$. In other words,

$$
\operatorname{dec}\left(\mathbf{M} \mathcal{A}^{\prime}(Z), R(\operatorname{cl}(\mathrm{b} \underline{\mathbf{M}} \mathcal{A}(Z)+\mathrm{b} \overline{\mathbf{M}} \mathcal{A}(Z)))>\aleph_{0} .\right.
$$

Proof. Let $\mathcal{N}^{\prime}$ be any subset of $Z$ homeomorphic to $\mathcal{N}([\mathrm{Ku}, 36, \mathrm{IV}$, Cor. 2]). Let $\varphi=\left(\varphi_{1}, \varphi_{2}, \ldots\right): \mathcal{N}^{\prime} \rightarrow{ }^{\mathbb{N}} \mathcal{N}$ be any homeomorphism. By Theorem 3.20 there exists a universal function $F \in \mathbf{M} \mathcal{A}^{\prime}(\mathcal{N} \times Z)$. Let $F_{n}(s, x)=F\left(\varphi_{n}(s), x\right)$ for $s \in \mathcal{N}^{\prime}$ and $x \in Z$. Then $F_{n} \in \mathbf{M} \mathcal{A}^{\prime}\left(\mathcal{N}^{\prime} \times Z\right)$ and thus $f_{n}: \mathcal{N}^{\prime} \rightarrow \mathbb{R}$ defined as $f_{n}(s)=F_{n}(s, s)$ belongs to $\mathbf{M} \mathcal{A}^{\prime}\left(\mathcal{N}^{\prime}\right)$ because, by our assumption on $\mathcal{A}, \mathcal{A}^{\prime}\left(\mathcal{N}^{\prime} \times \mathcal{N}^{\prime}\right)=\mathcal{A}^{\prime}\left(\mathcal{N}^{\prime} \times Z\right) \mid\left(\mathcal{N}^{\prime} \times \mathcal{N}^{\prime}\right)$. By Corollary 2.F and the fact that $\mathcal{A}^{\prime}\left(\mathcal{N}^{\prime}\right)=\mathcal{A}^{\prime}(Z) \mid \mathcal{N}^{\prime}$ and $\mathcal{N}^{\prime} \in(\mathcal{A}(Z))_{\delta}$, $f_{n}$ can be extended to a function $f_{n}^{*} \in \mathbf{M} \mathcal{A}^{\prime}(Z)$. By Theorem 2.A there exists $g \in \mathbf{M} \mathcal{A}^{\prime}(Z)$ such that $g(x) \neq f_{n}^{*}(x)$ for each $x \in Z$ and $n \in \mathbb{N}$.

Now assume that $g=\bigcup\left\{g_{n}: n \in \mathbb{N}\right\}$ and for each $n \in \mathbb{N}, g_{n} \in$ $\operatorname{cl}\left(\operatorname{bM}\left(\mathcal{A}(Z) \mid \operatorname{dom} g_{n}\right)+\mathrm{b} \overline{\mathbf{M}}\left(\mathcal{A}(Z) \mid \operatorname{dom} g_{n}\right)\right)$. By Lemma 3.19 each $g_{n}$ has an extension $g_{n}^{*} \in \operatorname{cl}(\mathrm{b} \underline{\mathbf{M}} \mathcal{A}(Z)+\mathrm{b} \overline{\mathbf{M}} \mathcal{A}(Z))$ for all $n \in \mathbb{N}$. There is an $s \in \mathcal{N}^{\prime}$ such that $F_{n}(s, x)=g_{n}^{*}(x)$ for each $n \in \mathbb{N}$. But then $f_{n}(s)=g_{n}(s)$ for each $n \in \mathbb{N}$ and, as $g(s) \in\left\{g_{n}(s): n \in \mathbb{N}\right\}$, we obtain $g(s)=f_{n_{0}}(s)$ for some $n_{0} \in \mathbb{N}$, which is a contradiction.

For any uncountable Polish space $Z$ we derive from Theorem 3.21 the following immediate corollaries.

Corollary 3.22 .

$$
\operatorname{dec}\left(\mathbf{B}_{\alpha+1}(Z), R\left(\operatorname{cl}\left(\mathrm{bL}_{\alpha}(Z)+\mathrm{b} \mathbf{U}_{\alpha}(Z)\right)\right)>\aleph_{0} .\right.
$$

Corollary 3.23 .

$$
\operatorname{dec}\left(\mathbf{M} \Sigma_{n+1}^{1}(Z), R\left(\operatorname{cl}\left(\mathrm{b} \underline{\mathbf{M}} \Sigma_{n}^{1}(Z)+\mathrm{b} \overline{\mathbf{M}} \Sigma_{n}^{1}(Z)\right)\right)>\aleph_{0} .\right.
$$

Acknowledgment. I wish to thank Professor Czesław Ryll-Nardzewski for his remarks concerning the subject of this paper.

I would also like to thank Professor Zbigniew Lipecki for his bibliographical remarks.

\section{References}

[CM] J. Cichoń and M. Morayne, Universal functions and generalized classes of functions, Proc. Amer. Math. Soc. 102 (1988), 83-89. 
[CMPS] J. Cichoń, M. Morayne, J. Pawlikowski and S. Solecki, Decomposing Baire functions, J. Symbolic Logic 56 (1991), 1273-1283.

[H] F. Hausdorff, Set Theory, Chelsea, New York 1962.

[Ke] S. Kempisty, Sur les séries itérées des fonctions continues, Fund. Math. 2 (1921), 64-73.

[Ku] K. Kuratowski, Topology I, Academic Press, New York 1966.

[L] A. Lindenbaum, Sur les superpositions de fonctions represéntables analytiquement, Fund. Math. 23 (1934), 15-37; Corrections, ibid., 304.

[Ma] R. D. Mauldin, On the Baire system generated by a linear lattice of functions, ibid. 68 (1970), 51-59.

[Mo] Y. N. Moschovakis, Descriptive Set Theory, North-Holland, Amsterdam 1980.

$\left[\mathrm{S}_{1}\right]$ W. Sierpiński, Sur les fonctions développables en séries absolument convergentes de fonctions continues, Fund. Math. 2 (1921), 15-27.

$\left[\mathrm{S}_{2}\right] \quad$ - Démonstration d'un théorème sur les fonctions de première classe, ibid., $37-40$.

INSTITUTE OF MATHEMATICS UNIWERSITY OF WROCEAW PL. GRUNWALDZKI $2 / 4$ 50-384 WROCŁAW, POLAND
Current address:

INSTITUTE OF MATHEMATICS POLISH ACADEMY OF SCIENCES ŚNIADECKICH 8 00-950 WARSZAWA, POLAND

Received 21 April 1991;

in revised form 3 March 1992 